\title{
CLASSIFICATION OF VERTEX-TRANSITIVE STRUCTURES
}

\author{
by \\ Stephanie Potter
}

\author{
A thesis \\ submitted in partial fulfillment \\ of the requirements for the degree of \\ Master of Science in Mathematics \\ Boise State University
}

May 2017 
(c) 2017

Stephanie Potter

ALL RIGHTS RESERVED 


\title{
BOISE STATE UNIVERSITY GRADUATE COLLEGE
}

\section{DEFENSE COMMITTEE AND FINAL READING APPROVALS}

\author{
of the thesis submitted by
}

\author{
Stephanie Potter
}

Dissertation Title: Classification of Vertex-Transitive Structures

Date of Final Oral Examination: 14 March 2017

The following individuals read and discussed the dissertation submitted by student Stephanie Potter, and they evaluated her presentation and response to questions during the final oral examination. They found that the student passed the final oral examination.

Samuel Coskey, Ph.D.

John Clemens, Ph.D.

Marion Scheepers, Ph.D.
Chair, Supervisory Committee

Member, Supervisory Committee

Member, Supervisory Committee

The final reading approval of the thesis was granted by Samuel Coskey, Ph.D., Chair of the Supervisory Committee. The thesis was approved by the Graduate College. 


\begin{abstract}
When one thinks of objects with a significant level of symmetry it is natural to expect there to be a simple classification. However, this leads to an interesting problem in that research has revealed the existence of highly symmetric objects which are very complex when considered within the framework of Borel complexity. The tension between these two seemingly contradictory notions leads to a wealth of natural questions which have yet to be answered.

Borel complexity theory is an area of logic where the relative complexities of classification problems are studied. Within this theory, we regard a classification problem as an equivalence relation on a Polish space. An example of such is the isomorphism relation on the class of countable groups. The notion of a Borel reduction allows one to compare complexities of various classification problems.

The central aim of this research is to determine the Borel complexities of various classes of vertex-transitive structures, or structures for which every pair or elements are equivalent under some element of its automorphism group. John Clemens has shown that the class of vertex-transitive graphs has maximum possible complexity, namely Borel completeness. On the other hand, we show that the class of vertextransitive linear orderings does not.

We explore this phenomenon further by considering other natural classes of vertextransitive structures such as tournaments and partial orderings. In doing so, we discover that several other complexities arise for classes of vertex-transitive structures.
\end{abstract}




\section{TABLE OF CONTENTS}

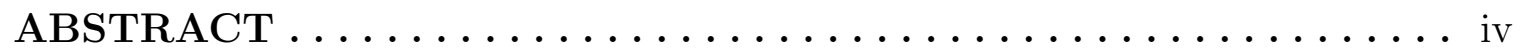

LIST OF FIGURES $\ldots \ldots \ldots \ldots \ldots \ldots \ldots \ldots \ldots \ldots$ vi

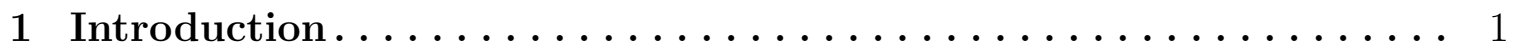

2 Vertex-Transitive Graphs and Partial Orders............ 8

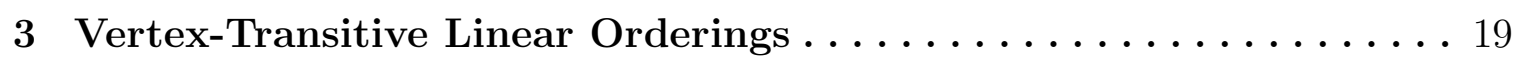

4 Vertex-Transitive Tournaments . . . . . . . . . . . . . . 29

REFERENCES.......................... 


\section{LIST OF FIGURES}

1.1 Hierarchy of relevant benchmark equivalence relations . . . . . . . . . . 3

1.2 Structures studied in this thesis $\ldots \ldots \ldots \ldots \ldots \ldots \ldots \ldots$

2.1 Depiction of $u_{i} R u_{j}$ with $u_{i}=g_{p} N$ and $u_{j}=g_{q} N \ldots \ldots \ldots \ldots \ldots$

2.2 Graph of $M$ as constructed by Mekler, $[8] \ldots \ldots \ldots \ldots \ldots \ldots$

2.3 Resulting square if there exist $k_{1}, k_{2} \in \mathbb{N}$ with $v_{k_{1}}$ adj $v_{k_{2}} \in G \ldots \ldots \ldots$

4.1 Directed edges from vertex $(0,0)$ to vertices one column to the right. . . 32 


\section{CHAPTER 1}

\section{INTRODUCTION}

Classification problems in Mathematics ask the question: "How can objects of a given type be identified and distinguished from one another, up to some equivalence relation?"

When one thinks of objects with a significant level of symmetry it is natural to expect there to be a simple classification. However, this leads to an interesting problem in that research has revealed the existence of highly symmetric objects which are very complex when considered within the framework of Borel complexity. The tension between these two seemingly contradictory notions leads to a wealth of natural questions which have yet to be answered. In this thesis we will answer some of these

questions in an attempt to further understand where vertex-transitive structures lie in terms of Borel complexity theory.

Borel complexity theory is an area of logic where the relative complexities of classification problems are studied. Within this theory, we regard a classification problem as an equivalence relation on a Polish space. An example of such is the isomorphism relation on the class of countable groups.

More generally, we can instead consider any invariant Borel class of countable structures, which we define below. 
Definition 1. Let $L=\left\{R_{i}: i \leq I\right\}$ be a finite relational language, where $R_{i}$ has arity $n_{i}$. Denote the space of all countable L-models as Mod $(L)$. Each element of $\operatorname{Mod}(L)$ can be viewed as an element of the product space

$$
X_{L}=\prod_{i \leq I} 2^{\mathbb{N}^{n_{i}}}
$$

That is, for every $x \in X_{L}$ let $M_{x} \in \operatorname{Mod}(L)$ be the countable model coded by $x$. Then for any $i \in I$ and $\left(k_{1}, \ldots, k_{n_{i}}\right) \in \mathbb{N}^{n_{i}}$, it is the case that $R_{i}^{M_{x}}\left(k_{1}, \ldots, k_{n_{i}}\right) \Leftrightarrow$ $x_{i}\left(k_{1}, \ldots, k_{n_{i}}\right)=1$. For the remainder of this paper we will identify $\operatorname{Mod}(L)$ and $X_{L}$.

Definition 2. The logic action of $S_{\infty}$ on $\operatorname{Mod}(L)$ is defined by letting $g \cdot M=N$ if and only if

$$
R_{i}^{N}\left(k_{1}, \ldots, k_{n_{i}}\right) \Leftrightarrow R_{i}^{M}\left(g^{-1}\left(k_{1}\right), \ldots, g^{-1}\left(k_{n_{i}}\right)\right)
$$

for all $i \in I$ and $\left(k_{1}, \ldots, k_{n_{i}}\right) \in \mathbb{N}^{n_{i}}$. Therefore, $g \cdot M=N$ if and only if $g$ is an isomorphism from $M$ onto $N$.

Definition 3. An invariant Borel class of countable L-structures is an $S_{\infty}$ invariant Borel subset of $\operatorname{Mod}(L)$.

Note that for the purposes of this thesis we always study the orbit equivalence relation, i.e. the isomorphism relation on either $\operatorname{Mod}(L)$ or on the invariant Borel class.

The notion of a Borel reduction allows one to compare complexities of various classification problems. 
Definition 4. Let $E$ and $F$ be equivalence relations on the Borel spaces $X$ and $Y$ respectively. $E$ is considered Borel reducible to $F$, denoted $E \leq_{B} F$, if there exists a Borel function $f: X \rightarrow Y$ such that, for $x, y \in X, x E y$ if and only if $f(x) F f(y)$.

$E$ and $F$ are bireducible to each other, denoted $E \sim_{B} F$, if both $E \leq_{B} F$ and $F \leq_{B} E$

Borel reducibility allows for organization of complexities into a hierarchy. The figure below shows the Borel reductions between the benchmark equivalence relations which are relevant to this thesis.

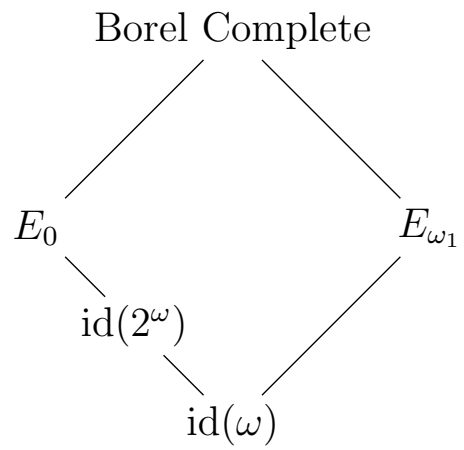

Figure 1.1: Hierarchy of relevant benchmark equivalence relations

These equivalence relations are increasing in the sense of Borel reducibility from bottom to top wherever there is an edge. We also note that there are many equivalence relations that lie in between those shown which are omitted for the purpose of readability.

At the "bottom" of the Borel hierarchy, we have $\operatorname{id}(\omega)$ which is defined as the equality relation on elements of $\omega$. While this equivalence relation will not be used, this gives one a good base as $\mathrm{id}(\omega)$ is the least complex among equivalence relations with infinitely many classes. 
We then see that there exists a Borel reduction from $\operatorname{id}(\omega)$ to $\operatorname{id}\left(2^{\omega}\right)$, or equality on an uncountable Polish space. Note that, for an equivalence relation $E$ on a set $X$, $E$ is said to be smooth if it is the case that $E$ is Borel reducible to id $\left(2^{\omega}\right)$.

In addition, we see that $\operatorname{id}(\omega)$ is less complex than $E_{\omega_{1}}$, or isomorphism on codes for countable ordinals.

Increasing in complexity, we arrive at the equivalence relation $E_{0}$ which is the immediate successor to $\operatorname{id}\left(2^{\omega}\right)$, among the Borel equivalence relations.

Definition 5. The equivalence relation $E_{0}$ is the relation of eventual equality on $2^{\omega}$. That is,

$$
x E_{0} y \Leftrightarrow \exists m \forall n \geq m x(n)=y(n) .
$$

At the "top" of our Borel hierarchy, we have Borel complete which is the maximum possible complexity among isomorphism problems for countable structures.

Definition 6. Given $C$ an invariant Borel class, $C$ is Borel complete if and only if, for every invariant Borel class $B, B$ is Borel reducible to $C$.

Some commonly referenced examples of Borel complete classes include the class of linear orders [3] and the class of directed graphs [4].

The central aim of this research is to determine the Borel complexities of vertextransitive structures. We say that a structure $A$ is vertex-transitive if, for every element $x, y \in A$, there exists an automorphism $\varphi$ of $A$ such that $\varphi(x)=y$. Interest in such structures stems from their notable symmetry. In this thesis, we explore the complexities of vertex-transitive graphs, partial orders, linear orders and tournaments whose relationships can be seen below. 


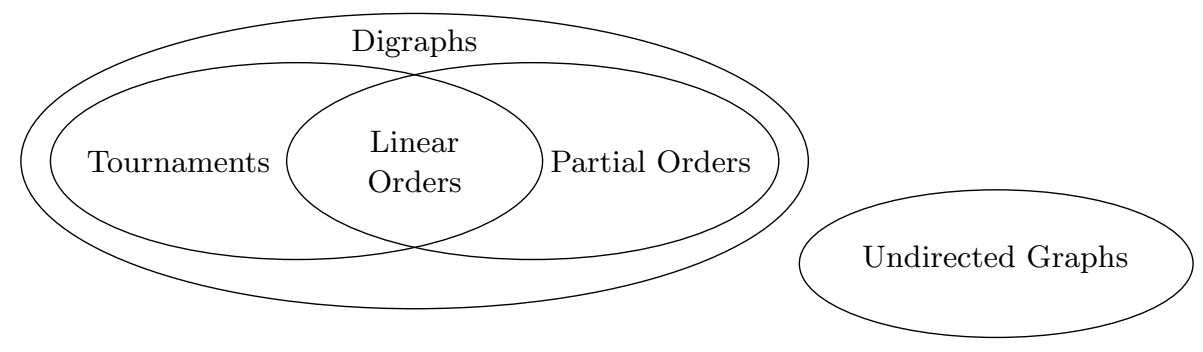

Figure 1.2: Structures studied in this thesis

It is important to remark that the class of vertex-transitive structures need not be Borel. To say that the isomorphism relation for such structures is Borel complete, it is sufficient to show that some Borel complete equivalence relation is Borel reducible to it. Formally, we give the following definitions of the structures seen in the figure above.

Definition 7. A graph is a pair of sets $(V, E)$ where $V$ is a set of vertices and $E$ is a set of edges formed by pairs of vertices in $V$. A directed graph, or digraph, is a graph in which edges are ordered pairs, where edge $(u, v)$ means that there is a directed edge from vertex $u$ to vertex $v$.

We note that, for our purposes, directed graphs have no self-loops and no bidirectional edges. Further, we see that all other structures can be realized as directed graphs. Narrowing our focus slightly, we find partial orders and tournaments.

Definition 8. A partial order is a binary relation $\leq$ on a set $X$ which is:

i. reflexive: $x \leq x$ for all $x \in X$

ii. anti-symmetric: $x \leq y$ and $y \leq x$ implies that $x=y$

iii. transitive: $x \leq y$ and $y \leq z$ implies $x \leq z$. 
Definition 9. A tournament is a directed graph in which every pair of distinct vertices is connected by a single directed edge.

Finally, tournaments which satisfy the properties of partial orders are linear orders, and vice versa.

Definition 10. A linear order is a partial order $\leq$ on a set $X$ which also satisfies the comparability axiom. That is, for every $x, y \in X$ either $x \leq y$ or $y \leq x$.

We begin by extending the results regarding vertex-transitive graphs in Clemens' paper titled Isomorphism of homogeneous structures [1]. In this paper, Clemens showed that the isomorphism relation on countable, connected, vertex-transitive, undirected graphs is Borel complete. Morover, he suggested that one could also prove this for the directed case. Here, we first cover the simpler directed case before filling in the missing components needed to prove the original result for undirected graphs. A consequence of this leads to the result that vertex-transitive partial orders are Borel complete.

This analysis then leads us to ask about the complexity of a special case of directed graphs: linear orders. We have shown that the class of vertex-transitive linear orderings is, in fact, not Borel complete. Further, we see that there exists an absolutely $\boldsymbol{\Delta}_{\mathbf{2}}^{\mathbf{1}}$ reduction from isomorphism on codes for countable ordinals, denoted $E_{\omega_{1}}$, to isomorphism on vertex-transitive linear orders.

We finish this paper by broadening our view slightly to consider vertex-transitive tournaments. That is, linear orders are a special type of tournament and so it is natural to see where tournaments lie in terms of Borel complexity. Here we show that isomorphism of vertex-transitive tournaments is properly more complex than $E_{0}$ however it remains a question as to whether or not this is Borel complete. 
Beyond the results given in this thesis it is natural to ask about other vertextransitive structures. For example, linear orders are a particular type of lattice, which are partial orders. Since we know that isomorphism of linear orders is Borel complete and isomorphism of partial orders is, in fact, Borel complete it is natural to ask if the class of vertex-transitive lattices is Borel complete as well.

Further, instead of vertex-transitive structures we could consider structures with larger automorphism groups. That is, an $n$-transitive structure is one in which its automorphism group acts transitively on $n$-tuples of distinct elements. So, we could instead ask the question: is the isomorphism problem for the class of $n$-transitive structures Borel complete for a given value $n$ ? 


\section{CHAPTER 2}

\section{VERTEX-TRANSITIVE GRAPHS AND PARTIAL ORDERS}

We begin by considering isomorphism of vertex-transitive graphs. The work shown here is an extension of results from Clemens's paper titled Isomorphism of homogeneous structures [1] in which he first showed that the isomorphism problem for countable, connected, vertex-transitive graphs is Borel complete.

The aim is to break up the proof that the isomorphism problems of countable connected vertex-transitive graphs is Borel complete into several components in order to uncover further results which follow from Clemens's ideas. We will first show that the class of extensional graphs is Borel complete. We then see that there exists a Borel reduction from the isomorphism relation on countable graphs to the isomorphism relation on countable, connected vertex-transitive graphs.

The original proof by Clemens focuses on the case where these graphs are undirected with a comment that we could instead consider the directed case given an additional edge restriction. Here, we first look at the directed case in which we form the directed Cayley graph of $H$, a group generated by the vertices of a countable graph, as directed Cayley graphs are always vertex-transitive. 
Definition 11. A connected graph is a graph in which there exists a path between every pair of vertices. A directed graph is weakly-connected if replacing all directed edges with undirected edges results in a connected graph.

Theorem 12 (Extracted from [1]). There exists a Borel reduction from countable graphs to countable, weakly-connected, vertex-transitive, directed graphs.

Proof. Let $\left\langle v_{i}\right\rangle_{i \in \mathbb{N}}$ enumerate the vertices of a countable graph $G$. Define $H$ to be the group generated freely by the the vertices of $G$ with the stipulation that adjacent vertices commute. That is, let $\mathbb{F}_{\omega}$ be the free group on generators $g_{i}$ and $N$ be the normal subgroup of $\mathbb{F}_{\omega}$ generated by $\left\{g_{i} g_{j} g_{i}^{-1} g_{j}^{-1}: v_{i}\right.$ adj $v_{j}$ in $\left.G\right\}$. Then, define $H=\mathbb{F}_{\omega} / N$ and form $\Gamma$, the directed Cayley graph of $H$ with generators $\left\langle g_{i}\right\rangle_{i \in \mathbb{N}}$. The vertices of $\Gamma$ are left cosets of $N$ in $\mathbb{F}_{\omega}$ and two vertices $w_{1} N$ and $w_{2} N$ are adjacent in $\Gamma$ if $g_{i} w_{1} N=w_{2} N$ with a directed edge from $w_{1} N$ to $w_{2} N$. Note that each of the generators $g_{i}$ are in distinct cosets.

In order to produce a code for this structure, we begin by fixing an enumeration $\left\langle w_{i}\right\rangle_{i \in \mathbb{N}}$ of words in $\mathbb{F}_{\omega}$. For each coset of $N$ we pick a representative for that coset by picking the least $i$ such that $w_{i}$ is in the given coset. This $w_{i}$ is the coset representative. Then, enumerate the chosen representatives and define the binary relation on $\mathbb{N}$ encoding $\Gamma$ according to whether corresponding cosets are adjacent in $\Gamma$.

We now aim to show that the map from $G$ to our code $\Gamma$ is the desired reduction from the isomorphism relation on countable graphs $G$ to the isomorphism relation on vertex-transitive, countable, weakly-connected, directed graphs. To see that $\Gamma$ is vertex transitive let $w_{1} N$ and $w_{2} N$ be vertices of $\Gamma$ and define the map 


$$
\varphi(w N)=w N w_{1}^{-1} w_{2}=w w_{1}^{-1} w_{2} N
$$

an automorphism of $\Gamma$ sending $w_{1} N$ to $w_{2} N$.

Now, suppose we have two isomorphic graphs $G_{1}$ and $G_{2}$ with $f$ an isomorphism between them. Let $N_{1}$ and $N_{2}$ be the normal subgroups in the construction of $\Gamma_{1}$ and $\Gamma_{2}$ respectively and let

$$
\varphi\left(w N_{1}\right)=\widetilde{w} N_{2}
$$

where, for $w=g_{i_{n}}^{\sigma_{n}} \ldots g_{i_{0}}^{\sigma_{0}}, \widetilde{w}=g_{f\left(i_{n}\right)}^{\sigma_{n}} \ldots g_{f\left(i_{0}\right)}^{\sigma_{0}}$. Well, $f$ induces a partial map $\varphi$ from their respective Cayley graphs,$\Gamma_{1}$ to $\Gamma_{2}$, such that $\varphi\left(g_{i} N_{1}\right)=g_{f(i) N_{2}}$ which acts on the cosets of the $g_{i}$. We then extend this partial map to an isomorphism on the graphs as a whole. Certainly, $\varphi$ is a bijection. To see that it is well-defined, note that the map taking $w$ to $\widetilde{w}$ is an automorphism of $\mathbb{F}_{w}$ which sends $N_{1}$ to $N_{2}$ and so $w_{1} w_{2}^{-1} \in N_{1}$ if and only if $\widetilde{w_{1}}{\widetilde{w_{2}}}^{-1} \in N_{2}$.

Now, suppose that $w_{1} N_{1} \rightarrow w_{2} N_{1}$ in $\Gamma_{1}$ or, in other words, $g_{k} w_{1} N_{1}=w_{2} N_{1}$. Then $\varphi$ gives that $\widetilde{\left(g_{k} w_{1}\right)} N_{2}=\widetilde{w_{2}} N_{2}$. Notice that $\widetilde{g_{k} w_{1}}=g_{f(k)} \widetilde{w_{1}}$ and so $g_{f(k)} \varphi\left(w_{1} N_{1}\right)=$ $\varphi\left(w_{2} N_{1}\right)$. The argument in the other direction is the same and so $w_{1} N_{1} \rightarrow w_{2} N_{1}$ in $\Gamma_{1}$ if and only if $\varphi\left(w_{1} N_{1}\right) \rightarrow \varphi\left(w_{2} N_{1}\right)$ in $\Gamma_{2}$.

Finally, suppose that $\Gamma_{1}$ and $\Gamma_{2}$ are isomorphic. We want to show that $G_{1} \cong G_{2}$ which can be achieved by seeing that one can recover $G$ (up to isomorphism) from the isomorphism class of $\Gamma$. Recalling that $\Gamma$ is vertex-transitive, without loss of generality we can fix a vertex in $\Gamma$ corresponding to $N$ and identify its adjacent vertices, $g_{k} N$. Enumerate these adjacent vertices as $\left\langle u_{i}\right\rangle_{i \in \mathbb{N}}$. Let $R$ be the binary relation on this set such that, for $u_{i} \neq u_{j}$, 


$$
u_{i} R u_{j} \Leftrightarrow \exists b \neq N \text { such that } u_{i}=g_{i} N \rightarrow b \text { and } u_{j}=g_{j} N \rightarrow b .
$$

The aim is to show that $u_{i} R u_{j}$ if and only if there exist $p, q \in \mathbb{N}$ with $u_{i}=g_{p} N$ and $u_{j}=g_{q} N$ such that $v_{p}$ adj $v_{q}$ in $G$. Well, if such a pair $p, q$ exists, then $g_{p}$ and $g_{q}$ commute in $H$. Then $u_{i}$ and $u_{j}$ are opposite corners of the square including $N$ and $g_{q} g_{p} N=g_{p} g_{q} N$. Thus $u_{i} R u_{j}$. Suppose now that $u_{i} R u_{j}$, with $u_{i} \neq u_{j}$. Let $u_{i}=g_{p} N$ and $u_{j}=g_{q} N$ with $N$ and $b=g_{n} g_{q} N=g_{m} g_{p} N$ as the other two vertices of the square.

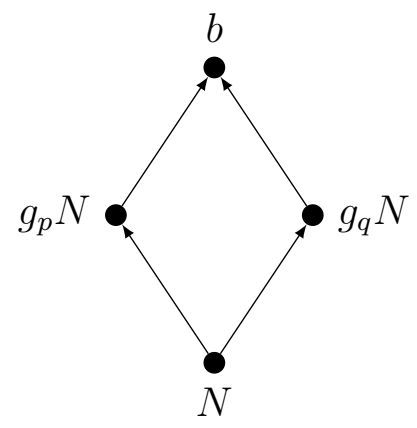

Figure 2.1: Depiction of $u_{i} R u_{j}$ with $u_{i}=g_{p} N$ and $u_{j}=g_{q} N$

Thus it must be that

$$
g_{p}^{-1} g_{m}^{-1} g_{n} g_{q} \in N
$$

Recalling that words in $N$ must have the sum of the exponents of each generator equal to 0 , it must be that $p=q$ and $m=n$ or $p=n$ and $m=q$. As $u_{i} \neq u_{j}$ then $g_{p} N \neq g_{q} N$ and so it cannot be the case that $p=q$ and $m=n$. Therefore, $g_{p}=g_{n}$ and $g_{m}=g_{q}$ and so

$$
g_{p}^{-1} g_{q}^{-1} g_{p} g_{q} \in N
$$


which means that $v_{p}$ adj $v_{q} \in G$. Thus we can recover from $\Gamma$ an isomorphic copy of $G$ by taking the out-neighbors of $N$ as the vertices and $R$ as the edge relation and so the desired Borel reduction exists.

With this, we have that the isomorphism relation on countable, connected, directed vertex-transitive graphs is Borel complete. The proof of the undirected case is very similar and so we exclude the redundancies. We also point out the additional cases required by not knowing whether the powers of the $g_{i}$ in equation (2.1) are 1 or -1 . Additionally, this proof requires that the graph $G$ be extensional.

Definition 13. An extensional graph is one in which, for any vertices $v_{1} \neq v_{2}$, there exists a third vertex $v_{3}$ such that $v_{1}$ adj $v_{3}$ and not $v_{2}$ adj $v_{3}$.

Fortunately, the following Lemma due to Mekler [8] tells us that the isomorphism relation on extensional graphs is also Borel complete. To prove this, note that the isomorphism relation of countable $\mathcal{L}_{0}$-structures is Borel complete [3], where $\mathcal{L}_{0}$ is the language containing a single binary relation symbol. We then show that there exists a Borel reduction from the isomorphism relation on $\mathcal{L}_{0}$-structures to extensional graphs which gives the desired conclusion.

Lemma 14 (Mekler). There exists a Borel reduction from the isomorphism relation on $\mathcal{L}_{0}$-structures to the isomorphism relation on extensional graphs. Thus, the class of extensional graphs is Borel complete.

Proof. Let $A$ be an $\mathcal{L}_{0^{-}}$-structure. First, build $G^{\prime}(A)$ such that its vertices are elements of $A$ and there are two nodes adjacent to each. If $A$ satisfies $a_{1} R a_{2}$, introduce two new vertices $\left(a_{1,2}\right)_{1}$ and $\left(a_{1,2}\right)_{2}$ such that $a_{1}$ adj $\left(a_{1,2}\right)_{1},\left(a_{1,2}\right)_{1}$ adj $\left(a_{1,2}\right)_{2}$, and $\left(a_{1,2}\right)_{2}$ adj $a_{2}$. Further, insert three new vertices adjacent to $\left(a_{1,2}\right)_{1}$ and four new vertices adjacent 
to $\left(a_{1,2}\right)_{2}$. The graph of $M$ constructed up to this point can be seen in the figure below.

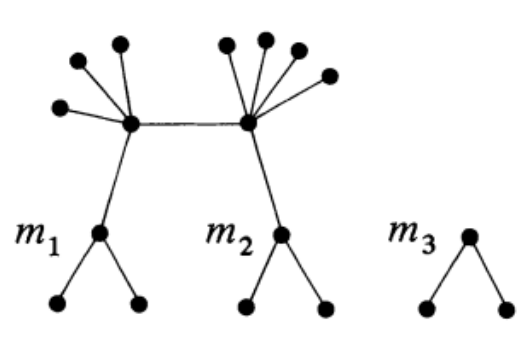

Figure 2.2: Graph of $M$ as constructed by Mekler, [8]

Finally, insert three new vertices adjacent to one another and to each of the previous vertices of $G^{\prime}(A)$. Once $G^{\prime}(A)$ is constructed, let each element of $G^{\prime}(A)$ be a vertex of $G(A) . G(A)$ is constructed by inserting a new vertex in the middle of each edge. Let $\left\langle v_{i}\right\rangle_{i \in \mathbb{N}}$ enumerate the vertices of $G(A)$. We note that $G(A)$ is extensional.

In order to show that this Borel map is, in fact, a Borel reduction we must show that $A_{1} \cong A_{2}$ if and only if $G\left(A_{1}\right) \cong G\left(A_{2}\right)$. If $A_{1} \cong A_{2}$, then there exists an isomorphism $\varphi: A_{1} \rightarrow A_{2}$ such that if $a_{1} R a_{2}$ in $A_{1}$ then $\varphi\left(a_{1}\right) R \varphi\left(a_{2}\right)$ in $A_{2}$. As $a_{1} R a_{2}$, we obtain the resulting encoding $G\left(A_{1}\right)$. Applying $\varphi$ to the vertices representing $a_{1}$ and $a_{2}$, we achieve an isomorphism $\varphi^{\prime}$ sending vertices in $G\left(A_{1}\right)$ to vertices in $G\left(A_{2}\right)$ and so $G\left(A_{1}\right) \cong G\left(A_{2}\right)$.

Suppose now that $G\left(A_{1}\right) \cong G\left(A_{2}\right)$. That is, there exists an isomorphism $\varphi^{\prime}: G\left(A_{1}\right) \rightarrow G\left(A_{2}\right)$ sending structures representing adjacent vertices in $A_{1}$ to structures representing adjacent vertices in $A_{2}$. Consider any pairs of elements $a_{1}$ adj $a_{2}$ in $A_{1}$ and $a_{3}$ adj $a_{4}$ in $A_{2}$. Then there exists an encoding in $G\left(A_{1}\right)$ of $a_{1}$ adj $a_{2}$ and similarly for $G\left(A_{2}\right)$. 
To see that we can recover the two vertices representing elements in $A_{1}$, one can locate all vertices which are a distance of two from vertices of degree four in $G(A)$. From these, one can identify $v_{1}$ and $v_{2}$ representing $a_{1}$ and $a_{2}$ respectively as follows: $v_{1}$ and $v_{2}$ have five vertices in between them, where $v_{1}$ is a distance of two from a vertex of degree eight and $v_{2}$ is a distance of two from a vertex of degree nine. Thus, by our encoding, we can recover that $a_{1}$ adj $a_{2}$.

As these graphs are isomorphic, we get that there exists an isomorphism $\varphi$ such that $v_{1} R v_{2}$ with respect to the encoding if and only if $\varphi\left(v_{1}\right) R \varphi\left(v_{2}\right)$ or, equivalently, $v_{3} R v_{4}$. Thus, we arrive at $a_{1}$ adj $a_{2}$ if, and only if $a_{3}$ adj $a_{4}$ and so $A_{1} \cong A_{2}$ as expected.

Theorem 15 (Clemens). There exists a Borel reduction from extensional graphs $G$ to countable, connected, undirected, vertex-transitive graphs.

Proof. The proof of this result very closely parallels the proof of Theorem 12, except we form $\Gamma$, the undirected Cayley graph of $H$, where two vertices $w_{1} N$ and $w_{2} N$ are adjacent in $\Gamma$ if there exists a generator $g_{i}$ such that $g_{i} w_{1} N=w_{2} N$ or $g_{i} w_{2} N=w_{1} N$. We then continue to show that the map $G \mapsto \Gamma$ is the desired reduction. Showing that $G_{1} \cong G_{2}$ implies $\Gamma_{1} \cong \Gamma_{2}$ is the same as in the proof of Theorem 12 .

In order to see that $\Gamma_{1} \cong \Gamma_{2}$ gives $G_{1} \cong G_{2}$ requires showing that $G$ can be recovered from $\Gamma$. This becomes slightly more involved than in the directed case. The argument begins the same; however, instead of the definition of the relation $R$ given above, for two distinct elements $u_{i}$ and $u_{j}$ in $\Gamma$ we define $R$ as

$$
u_{i} R u_{j} \Leftrightarrow \exists a \exists b, a \neq b, \text { such that } u_{i} \text { and } u_{j} \text { are each adjacent to both } a \text { and } b
$$


We then aim to show that $u_{i} R u_{j}$ if and only if there exist $k_{1}, k_{2} \in \mathbb{N}$ and $\sigma_{1}, \sigma_{2} \in$ $\{-1,1\}$ with $u_{i}=g_{k_{1}}^{\sigma_{1}} N$ and $u_{j}=g_{k_{2}}^{\sigma_{2}} N$ such that $v_{k_{1}}$ adj $v_{k_{2}}$ in $G$.

Well, if such a pair $k_{1}, k_{2}$ exists then $g_{k_{1}}$ and $g_{k_{2}}$ commute in $H$. Thus $u_{i}$ and $u_{j}$ are at opposite corners of a square shared with $N$ and $g_{k_{1}}^{\sigma_{1}} g_{k_{2}}^{\sigma_{2}} N=g_{k_{2}}^{\sigma_{2}} g_{k_{1}}^{\sigma_{1}} N$ and so $u_{i} R u_{j}$

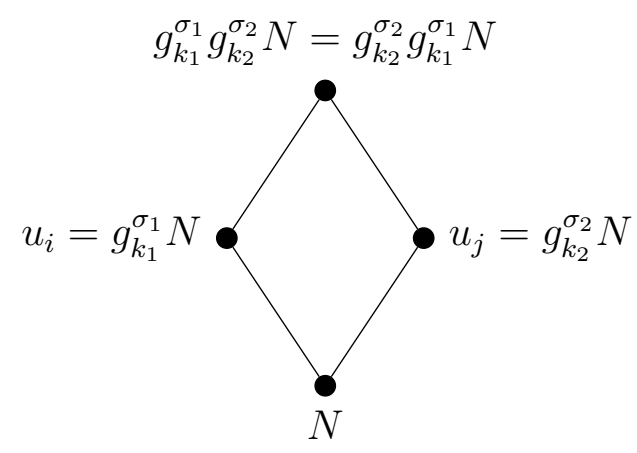

Figure 2.3: Resulting square if there exist $k_{1}, k_{2} \in \mathbb{N}$ with $v_{k_{1}}$ adj $v_{k_{2}} \in G$

Now, suppose that $u_{i} R u_{j}$ with $u_{i}=g_{k_{1}}^{\sigma_{1}} N$ and $u_{j}=g_{k_{2}}^{\sigma_{2}} N$ and $a, b$ the other two vertices of the square. Thus there must exist generators $g_{n_{1}}, g_{n_{2}}, g_{m_{1}}, g_{m_{2}}$ and $\tau_{1}, \tau_{2}, \rho_{1}, \rho_{2} \in\{-1,1\}$ such that

$$
a=g_{n_{1}}^{\tau_{1}} g_{k_{1}}^{\sigma_{1}} N=g_{n_{2}}^{\tau_{2}} g_{k_{2}}^{\sigma_{2}} N \text { and } b=g_{m_{1}}^{\rho_{1}} g_{k_{1}}^{\sigma_{1}} N=g_{m_{2}}^{\rho_{2}} g_{k_{2}}^{\sigma_{2}} N
$$

So we have

$$
g_{k_{1}}^{-\sigma_{1}} g_{n_{1}}^{-\tau_{1}} g_{n_{2}}^{\tau_{2}} g_{k_{2}}^{\sigma_{2}} \in N \text { and } g_{k_{1}}^{-\sigma_{1}} g_{m_{1}}^{-\rho_{1}} g_{m_{2}}^{\rho_{2}} g_{k_{2}}^{\sigma_{2}} \in N
$$


As words in $N$ must have the sum of the exponents of each generator equal to 0 , then it must be that $k_{1}=k_{2}, k_{1}=n_{1}$, or $k_{1}=n_{2}$. If $k_{1}=k_{2}$ then it must be that $-\sigma_{1}=\sigma_{2}$. Otherwise, $u_{i}=g_{k_{1}}^{\sigma_{1}} N$ and $u_{j}=g_{k_{2}}^{\sigma_{2}} N$ would be the same. Further, this would require that $n_{1}=n_{2}=k_{1}=k_{2}$ and so $\sigma_{2}-\sigma_{1}+\tau_{2}-\tau_{1}=0$. Well, then we would need $-\sigma_{1}=\tau_{1}$ and so $a=g_{k_{1}}^{-\sigma_{1}} g_{k_{1}}^{\sigma_{1}} N=N$. In the case that $k_{1} \neq k_{2}$ and $k_{1}=n_{1}$, we get that $-\sigma_{1}=\tau_{1}$ and so, once again, $a=g_{k_{1}}^{-\sigma_{1}} g_{k_{1}}^{\sigma_{1}} N=N$. Finally, for the case where $k_{1} \neq k_{2}$ and $k_{1}=n_{2}$ it also must be the case that $k_{2}=n_{1}, \sigma_{1}=\tau_{2}$, and $\sigma_{2}=\tau_{1}$. Well, then $g_{k_{1}}^{-\sigma_{1}} g_{k_{2}}^{-\sigma_{2}} g_{k_{1}}^{\sigma_{1}} g_{k_{2}}^{\sigma_{2}} \in N$ and so $v_{k_{1}}$ adj $v_{k_{2}}$ in $G$.

Repeating the same argument for $b$, we see that either $b=N$ or $v_{k_{1}}$ adj $v_{k_{2}}$ in $G$. Recalling that $a \neq b$ it must be the case that $v_{k_{1}}$ adj $v_{k_{2}}$ in $G$. Thus we can identify pairs of elements $\left\{u_{i}, u_{j}\right\}$, so that they are $R$-related to all of the same elements. That is, we have identified pairs $\left\{g_{k} N, g_{k}^{-1} N\right\}$. By extensionality, this will not identify any other pairs as, given $g_{i}$ and $g_{j}, i \neq j$, there exists a $g_{k}$ which commutes with $g_{i}$ but not with $g_{j}$ and vice versa.

Therefore, we form the graph with these pairs as its vertices where two vertices are set adjacent if each element in the first pair is $R$-related to each element in the second pair. The relation $R$ then gives that this graph is isomorphic to $G$.

Therefore, the isomorphism relation on countable, connected, vertex-transitive graphs is Borel complete. A further consequence is that vertex-transitive partial orders are also Borel complete. This can be seen by taking the transitive closure of the directed graph from Theorem 12 which will result in a partial order, as we will do below. First, we recall the definition of a partial order:

Definition 16. A partial order is a relation which is reflexive, antisymmetric, and transitive. 
Definition 17. The transitive closure of a directed graph $G$, denoted $C(G)$, is a graph which contains an edge $\{u, v\}$ whenever there is a directed path from u to $v$, for $u, v \in G$ as well as a path of length 0 from $u$ to $u$.

It is important to note that the transitive closure of a directed graph does not always yield a partial order. For this to be the case, it must be that the directed graph has no cycles. For the following statement, we remind the reader that, in this paper, directed graphs have no cycles of length one or two.

Proposition 18. If $G$ a directed graph, then the transitive closure $C(G)$, is a partial order if, and only if, G has no directed cycles.

Proof. For the forward direction, suppose $C(G)$ satisfies the requirements of a partial order. Further, suppose towards a contradiction that $G$ contains a cycle such that $x_{1} \leq x_{2} \leq \cdots \leq x_{n} \leq x_{1}$ for $x_{1}, x_{2}, \ldots, x_{n}$ vertices of $G(A)$. However, as $C(G)$ is antisymmetric, $x_{1} \leq x_{i}$ and $x_{i} \leq x_{1}$ gives $x_{1}=x_{i}$ for $1 \leq i \leq n$. Therefore, it cannot be the case that $G$ has a cycle.

Now, suppose there exist no cycles in $G$. To show that $C(G)$ satisfies the requirements of a partial order, we first note that the transitive closure is, in fact, transitive. Further, we assumed the relation on $A$ was reflexive and so $C(G)$ remains transitive, as this does not affect single vertices but the relationship between two vertices. And finally, as there are no cycles in $G$ it must be that there are no cycles in $C(G)$ and so we achieve antisymmetry. Thus the transitive closure of $G$ admits a partial order.

Corollary 19. There exists a Borel reduction from the isomorphism relation on extensional graphs $G$ to the isomorphism relation on countable vertex-transitive partial orders. 
Proof. Recalling Proposition 18, note that the directed graph $\Gamma$ of Theorem 12 has no directed cycles and so the transitive closure, $C(\Gamma)$, is a partial order. To see that $\Gamma$ has no directed cycles, note that $\Gamma$ is the directed Cayley graph of $H$ in which, for all words $w=w_{1} \ldots w_{k}$ having generators $g_{1}, \ldots, g_{k}$, if $w=1$ in $H$ then the sum of all exponents of each of the corresponding $g_{i}$ must equal 0 . Therefore, the sum is not positive and so there cannot be a directed cycle.

Therefore, given $\Gamma$ one can produce its transitive closure, $C(\Gamma)$, which is a partial order. The aim is to show that $\Gamma_{1} \cong \Gamma_{2}$ if and only if $C\left(\Gamma_{1}\right) \cong C\left(\Gamma_{2}\right)$. If $\Gamma_{1} \cong \Gamma_{2}$ then we certainly have that $C\left(\Gamma_{1}\right) \cong C\left(\Gamma_{2}\right)$ as transitive closure is isomorphism invariant. So, now suppose that $C\left(\Gamma_{1}\right) \cong C\left(\Gamma_{2}\right)$. Given $C(\Gamma)$ we want to show that we can recover the original $b \in \Gamma$ such that $N \rightarrow b$ in $\Gamma$ from any point $p \in C(\Gamma)$.

The claim is that the out-neighbors of $N$ are all of the $b \in \Gamma$ such that $N \rightarrow b$ in $C(\Gamma)$ and there does not exist a directed path of length greater than one from $N$ to $b$ in $C(\Gamma)$. This is the case as, if there did exist such a path in $C(\Gamma)$ then we would have

$$
b=g_{j} N \text { and } b=g_{i_{1}} \ldots g_{i_{n}} N
$$

which would mean that $g_{i_{1}} \ldots g_{i_{n}} g_{j}^{-1}=1$ in $H$ which contradicts that words in $N$ must have the sum of the exponents of all generators equal to 0 . Thus we have recovered $\Gamma$ from $C(\Gamma)$ and so there exists a Borel reduction from extensional graphs to countable partial orders. 


\section{CHAPTER 3}

\section{VERTEX-TRANSITIVE LINEAR ORDERINGS}

After classifying directed graphs in the previous chapter it is natural to ask about the complexity of special cases of directed graphs such as linear orders. The main theorem of this chapter isolates the complexity of vertex-transitive linear orders, in particular. Before presenting this result, it is necessary to discuss the condensation of linear orders. Condensation will be used in the main theorem in order to classify vertex-transitive linear orders by countable ordinals (refer to [9] for more details).

First, let us recall that a linear order is a partial order $\leq$ on a set $X$ which also satisfies comparability: for every $x, y \in X$, either $x \leq y$ or $y \leq x$.

Definition 20. Let $L$ be a linear ordering and let $L^{\prime}$ be a collection of non-empty intervals of $L$ which partitions $L$, ordered by

$$
I_{1} \ll I_{2} \text { if, for all } x_{1} \in I_{1}, x_{2} \in I_{2}, x_{1}<x_{2} \text {. }
$$

$L^{\prime}$ is called a condensation of $L$.

Intuitively, we think of the condensation as whole intervals of $L$ being condensed to single points in $L^{\prime}$. It is also important to note that the condensation of a vertextransitive linear ordering remains vertex-transitive. That is, if $L$ is vertex-transitive then there exists an automorphism $\varphi$ such that $\varphi(x)=y$ for every pair $x, y \in L$ 
and this automorphism extends to $C[L]$. For our purposes, we will make use of finite condensation maps, where discrete intervals are condensed.

Definition 21. The finite condensation map of $L$ is denoted

$$
c_{F}(x)=\{y \mid[x, y] \text { or }[y, x] \text { is finite }\}
$$

for $x \in L$. Let $c_{F}[L]$ denote the condensation of $L$ determined by the intervals $c_{F}(x)$ for all $x \in L$.

Note that $c_{F}(x)$ can be finite or, otherwise, has order type $\omega, \omega^{*}$ or $\mathbb{Z}$. We call $c_{F}(x)$ the equivalence class of $x$. This condensation map will be used implicitly throughout this chapter. Considering more than a single condensation leads to the following iterative definitions.

Definition 22. We define an iterated condensation map with $c^{1}=c$ as

(i) $c^{\alpha+1}(x)=\left\{y \mid c\left(c^{\alpha}(x)\right)=c\left(c^{\alpha}(y)\right)\right\}$

(ii) Assume that $\lambda$ is a limit ordinal and that for each $\beta<\lambda$ we have defined for each linear ordering $A$ the condensation map $c^{\beta}$. Then we define the condensation map $c^{\lambda}$ by

$$
c^{\lambda}(x)=\bigcup\left\{c^{\beta}(x) \mid \beta<\lambda\right\}
$$

With the above definition of iterated condensations we note that, given a linear order $L$, the condensation map stabilizes after a bounded number of iterations. The following proposition shows that this is, in fact, the case. 
Proposition 23. Let $L$ be a linear ordering of cardinality $\kappa$. Then there exists an ordinal $\alpha<\kappa^{+}$such that $c^{\beta}(x)=c^{\alpha}(x)$ for all $x \in L$ and for all $\beta \geq \alpha$.

Proof. If $c^{\alpha+1}(x)=c^{\alpha}(x)$ for every $x \in L$, then $c^{\beta}(x)=c^{\alpha}(x)$ for every $\beta \geq \alpha$. Thus, for some set $S=\left\{\alpha \mid c^{\alpha+1}(x) \neq c^{\alpha}(x)\right.$ for some $\left.x \in L\right\}, S$ is an initial segment of ordinals.

Further, for each $\alpha \in S$, there exists a pair $\{x, y\}$ of elements of $L$ such that $c^{\alpha}(x) \neq c^{\alpha}(y)$ but $c^{\alpha+1}(x)=c^{\alpha+1}(y)$. So $S$ is an initial segment of the ordinals which is in a one-to-one correspondence with a subset of $L \times L$. Thus $S$ has cardinality at most $\kappa$.

Therefore, $\alpha$ has cardinality $\kappa$ and so $\kappa \leq \alpha \leq \kappa^{+}[9]$. Thus we have an $\alpha<\kappa^{+}$ such that $c^{\beta}(x)=c^{\alpha}(x)$ for every $x \in L$ and for every $\beta \geq \alpha$.

Recalling that our aim is to understand vertex-transitive linear orders, we will show that there are two such classes of vertex-transitive linear orders, namely powers of $\mathbb{Z}$ and $\mathbb{Q}$ copies of powers of $\mathbb{Z}$. This leads us to the following definition:

Definition 24. Given an ordinal $\beta, \mathbb{Z}_{0}(\beta)$ consists of all $\beta$-sequences of elements of $\mathbb{Z}$ which have only finitely many non-zero entries. That is

$$
\mathbb{Z}_{0}(\beta)=\{s: \beta \rightarrow \mathbb{Z} \mid\{\alpha: s(\alpha) \neq 0\} \text { is finite }\}
$$

ordered by $s \ll t$ if $s(\mu)<t(\mu)$ where $\mu$ is the largest ordinal $\gamma<\beta$ for which $s(\gamma) \neq t(\gamma)$ 
For a more intuitive definition of powers of $\mathbb{Z}$, one can recursively construct a definition of $\mathbb{Z}_{0}(\beta)$, which we will call $\mathbb{Z}^{\beta}$, as follows:

\section{Definition 25. (i) $\mathbb{Z}^{0}=1$}

(ii) $\mathbb{Z}^{\beta+1}=\mathbb{Z}^{\beta} \cdot \omega^{*}+\mathbb{Z}^{\beta}+\mathbb{Z}^{\beta} \cdot \omega=\mathbb{Z}^{\beta} \cdot \mathbb{Z}$

(iii) $\mathbb{Z}^{\lambda}=\left(\sum\left\{\mathbb{Z}^{\gamma} \cdot \omega \mid \gamma<\lambda\right\}\right)^{*}+1+\sum\left\{\mathbb{Z}^{\gamma} \cdot \omega \mid \gamma<\lambda\right\}$ for limit ordinals $\lambda$.

Note that we define the sum of arbitrarily many linear orderings as follows. Let $\langle I, R\rangle$ be a linear ordering and, for each $i \in I$, let $\left\langle A_{i}, S_{i}\right\rangle$ be a linear ordering. Then $\sum_{i \in I} A_{i}$ is defined to be the linear ordering $\langle C, T\rangle$ with $C=\bigcup_{i \in I} A_{i}$ and, for any two $c_{1}, c_{2} \in C$

$$
\begin{aligned}
c_{1}<_{T} c_{2} \text { if } \quad & \left(c_{1} \in A_{i} \text { and } c_{2} \in A_{j} \text { and } i<_{R} j\right) \text { or } \\
& \left(c_{1} \in A_{i} \text { and } c_{2} \in A_{i} \text { and } c_{1}<_{S_{i}} c_{2} \text { for some } i \in I\right) .
\end{aligned}
$$

Further, we define the product of two linear orders, $\langle I, R\rangle$ and $\langle A, S\rangle$ as

$$
A \cdot I=\sum_{i \in I} A
$$

where addition is as defined above.

The following proposition tells us that the previous definitions of powers of $\mathbb{Z}$ are, in fact, equivalent.

Proposition 26. For any ordinal $\beta, \mathbb{Z}_{0}(\beta) \cong \mathbb{Z}^{\beta}$.

Proof. This is proved by induction on $\beta$. First, consider the case $\mathbb{Z}_{0}(0) \cong \mathbb{Z}^{0}$. By definition $\mathbb{Z}^{0}=1$ and so now $\mathbb{Z}_{0}(0)=\{s: 0 \rightarrow \mathbb{Z} \mid \exists N \forall n \geq N s(n)=0\} \cong\{\emptyset\} \cong 1$. Thus $\mathbb{Z}^{0}=1 \cong \mathbb{Z}_{0}(0)$. 
Now, suppose we have that $\mathbb{Z}_{0}(\beta)=\mathbb{Z}^{\beta}$ and let us show that the successor case holds, i.e. $\mathbb{Z}_{0}(\beta+1) \cong \mathbb{Z}^{\beta+1}$ below:

$$
\begin{aligned}
\mathbb{Z}^{\beta+1} & =\mathbb{Z}^{\beta} \cdot \mathbb{Z} \\
& \cong \mathbb{Z}_{0}(\beta) \cdot \mathbb{Z}(\text { by assumption }) \\
& \cong\{(s:(\beta) \rightarrow \mathbb{Z}, t \in \mathbb{Z}) \mid \exists N \forall n \geq N s(n)=0\} \\
& =\mathbb{Z}_{0}(\beta+1) .
\end{aligned}
$$

Finally, for a limit ordinal $\lambda$, we claim that $\mathbb{Z}_{0}(\lambda)=\{s: \lambda \rightarrow \mathbb{Z} \mid\{\alpha: s(\alpha) \neq 0\}$ is finite $\}$ and $\mathbb{Z}^{\lambda}=\left(\sum\left\{\mathbb{Z}^{\gamma} \cdot \omega \mid \gamma<\lambda\right\}\right)^{*}+1+\sum\left\{\mathbb{Z}^{\gamma} \cdot \omega \mid \gamma<\lambda\right\}$ are isomorphic.

To see this, let $\oslash \in \mathbb{Z}_{0}(\lambda)$ denote the sequence $s(\alpha)=0$ for all $\alpha$. This $\oslash$ corresponds to the " 1 " in the definition of $\mathbb{Z}^{\lambda}$. Next, the $s \in \mathbb{Z}_{0}(\lambda)$ such that $s(0)>0$ and $s(\alpha)=0$ for all $\alpha>0$ are a copy of $\mathbb{Z}^{0} \cdot \omega=\omega$.

In general, the elements $s \in \mathbb{Z}_{0}(\lambda)$ with $s(\gamma)>0$ and $s(\alpha)=0$ for all $\alpha>\gamma$ are a copy of $\mathbb{Z}^{\gamma} \cdot \omega$. Thus, we account for the sum $\sum\left\{\mathbb{Z}^{\gamma} \cdot \omega \mid \gamma<\lambda\right\}$ in the definition of $\mathbb{Z}^{\lambda}$.

There is an analogous correspondence between sequences in $\mathbb{Z}_{0}(\lambda)$ and the sum $\left(\sum\left\{\mathbb{Z}^{\gamma} \cdot \omega \mid \gamma<\lambda\right\}\right)^{*}$. Thus, $\mathbb{Z}_{0}(\lambda) \cong \mathbb{Z}^{\lambda}$ for $\lambda$ a limit ordinal.

Therefore we conclude that, for any ordinal $\beta, \mathbb{Z}_{0}(\beta) \cong \mathbb{Z}^{\beta}$.

To understand how condensations of power of $\mathbb{Z}$ behave, let us first look at a single condensation of $\mathbb{Z}^{\beta+1}$ where $\beta+1$ is finite. 
Proposition 27. A single condensation of $\mathbb{Z}^{\beta+1}$, for $\beta+1<\omega$, reduces to $\mathbb{Z}^{\beta}$.

Proof. By definition of finite condensation, we see that

$$
c\left[\mathbb{Z}^{\beta+1}\right]=\left\{\{(a, b) \mid b \in \mathbb{Z}\} \mid a \in \mathbb{Z}^{\beta}\right\} \cong \mathbb{Z}^{\beta}
$$

and so a single condensation of $\mathbb{Z}^{\beta+1}$ reduces to $\mathbb{Z}^{\beta}$ in the case that $\beta+1<\omega$.

Moreover, given sequences in $\mathbb{Z}^{\lambda}$ for some limit ordinal $\lambda$ we see that condensations of such sequences behave as described in the following proposition.

Proposition 28. For $\mathbb{Z}^{\lambda}=\{s: \lambda \rightarrow \mathbb{Z} \mid\{\alpha: s(\alpha) \neq 0\}$ is finite $\}$, given two sequences $s, t \in \mathbb{Z}^{\lambda}$

(i) $c(s)=\left\{t \mid s \uparrow_{[1, \lambda)}=t \uparrow_{[1, \lambda)}\right\}$

(ii) $c^{\gamma}(s)=\left\{t \mid s \uparrow_{[\gamma, \lambda)}=t \uparrow_{[\gamma, \lambda)}\right\}$ for each ordinal $\gamma$.

Proof. Let $\mathbb{Z}^{\lambda}=\{s: \lambda \rightarrow \mathbb{Z} \mid\{\alpha: s(\alpha) \neq 0\}$ is finite $\}$ and consider two sequences $s, t \in \mathbb{Z}^{\lambda}$.

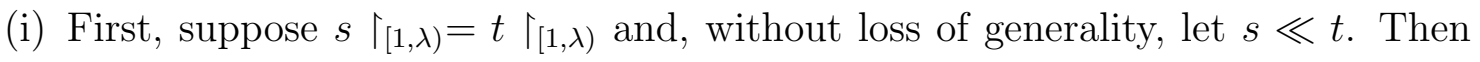
we must have $s(0)<t(0)$. If there exists a third $r \in \mathbb{Z}^{\lambda}$ and $r$ differs at a latter point $\gamma$ then it does from both $s$ and $t$ so either $r \ll s \ll t$ or $s \ll t \ll r$. If $s \ll r \ll t$ then it must be that $s(0)<r(0)<t(0)$ and there are only finitely many $r$ such that this is the case. Therefore $[s, t]$ is finite and so $c(s)=c(t)$.

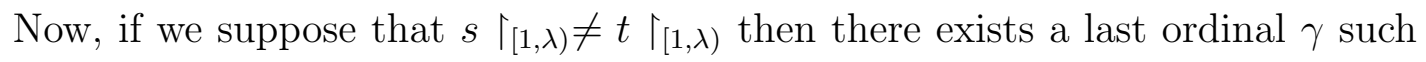
that $1 \leq \gamma<\lambda$ where $s(\gamma) \neq t(\gamma)$. Without loss of generality, let $s(\gamma)<t(\gamma)$. Let $r_{n} \in \mathbb{Z}^{\lambda}$ be defined as $r_{n}=n \frown s \uparrow_{[1, \lambda)}$ for any $n \in \mathbb{Z}$. Then $s \ll r_{n} \ll t$ for 
$n>s(0)$ and there are infinitely many $r_{n}$ which satisfy this. Therefore, $[s, t]$ is infinite and so $c(s) \neq c(t)$.

Therefore, we conclude that $c(s)=\left\{t \mid s \uparrow_{[1, \lambda)}=t \uparrow_{[1, \lambda)}\right\}$.

(ii) This case follows using a similar argument to (i) and induction on $\gamma$.

As we alluded to earlier in the chapter, there are two classes of vertex-transitive linear orders. In order to prove this, we require the following definitions about single elements of $L$.

Definition 29. Given a linear ordering $L$,

(i) $x \in L$ is left dense if $x$ is not the least element in $L$ and if there is no greatest element $y<x$

(ii) $x \in L$ is right dense if $x$ is not the greatest element in $L$ and if there is no least element $y>x$

(iii) $x \in L$ is left discrete if there exists a greatest $y \in L$ such that $y<x$ or if $x$ is the greatest element of $L$

(iv) $x \in L$ is right discrete if there exists a least $y \in L$ such that $y>x$ or if $x$ is the least element of $L$.

Note that, given some element $x \in L, x$ will be either left/right dense or left/right discrete. This leads us to the following lemma, where we will see that $\mathbb{Z}^{\beta}$ and $\mathbb{Z}^{\beta} \cdot \mathbb{Q}$, for countable ordinals $\beta$, (along with the trivial linear order 1 ) are the only possible classes of vertex-transitive linear orders. 
Lemma 30. If a linear ordering $L$ is vertex-transitive, then

(i) if one point is left or right dense then every point is and, in fact, $L \cong \mathbb{Q}$

(ii) if one point is left or right discrete then every point is, and every equivalence class is a copy of the integers or $L=1$.

Proof. By vertex-transitivity, if one point is left or right dense then every point must also be left or right dense, respectively.

(i) First, let $a<b$. If $b$ is left dense then, given a sequence $\left\{b_{i}\right\}_{i \in \mathbb{N}}$ approaching $b$ from the left, there must eventually be some $n \in \mathbb{N}$ such that $a<b_{n}<b$. Therefore, $L$ is dense. Similarly, let $b<a$ be right dense. Then, given a sequence $\left\{b_{i}\right\}_{i \in \mathbb{N}}$ approaching $b$ from the right, there must eventually be some $n \in \mathbb{N}$ such that $b<b_{n}<a$. Once again, $L$ is dense.

As $L$ is a dense linear ordering, then $L$ is isomorphic to $\mathbb{Q}, \mathbb{Q} \cup\{\infty\},\{-\infty\} \cup \mathbb{Q}$, or $\{-\infty\} \cup \mathbb{Q} \cup\{\infty\}$. However, as $L$ is also vertex-transitive there can be no first/last element and so it must be the case that $L \cong \mathbb{Q}$.

(ii) Similar to above, vertex-transitivity tells us that if one point is left or right discrete then every point must be left or right discrete. Thus $L$ is discrete and so every equivalence class is either a single point, a finite number of points, $\omega$, $\omega^{*}$, or $\mathbb{Z}$. As before, the vertex-transitivity of $L$ requires that there exists no first/last element of any class and so we must have that every equivalence class is a copy of the integers in the case that $L$ has more than one element.

Following Proposition 23, which states that we reach a condensation fixed-point after a bounded number of iterations, and the discussion of iterated condensations of 
vertex-transitive linear orders, it is natural to ask what happens when we reach such a fixed-point.

Proposition 31. Given a vertex-transitive linear order $L$, if $c(x)=\{x\}$ for every $x \in L$ then $L$ is isomorphic to either $\mathbb{Q}$ or 1 .

Proof. Let $L$ be a vertex-transitive linear order and $c(x)=\{x\}$ for every $x \in L$. There are two cases to consider:

(i) If one point is left/right dense then, by Lemma $30, L \cong \mathbb{Q}$.

(ii) If one point is left/right discrete then Lemma 30 tells us that there are two possibilities. The first is that $L=1$. Otherwise, every equivalence class of $L$ is a copy of the integers. However, if this is the case then, for any $x \in L$, $c(x)=\{y \mid[x, y]$ or $[y, x]$ is finite $\} \neq\{x\}$ as every $x \in \mathbb{Z}$ is a finite distance from at least one other $y \in \mathbb{Z}$. Thus we reach a contradiction and so if one point is left/right discrete at $c(x)=\{x\}$ for every $x \in L$ then $L=1$.

By Proposition 23 we are able to conclude that for countable, vertex-transitive linear orders it takes countably many steps to arrive at a condensation fixed point. Further, by Proposition 31 the possible condensation fixed points are either 1 or $\mathbb{Q}$. In summary, we arrive at the following theorem.

Theorem 32. If $L$ is a countable vertex-transitive linear order then $L$ is isomorphic to either $\mathbb{Z}^{\beta}$ or $\mathbb{Z}^{\beta} \cdot \mathbb{Q}$ for some $\beta<\omega_{1}$.

To conclude this chapter, we find that there exists an absolutely $\boldsymbol{\Delta}_{2}^{1}$ reduction from isomorphism of codes for countable ordinals to isomorphism of vertex-transitive linear orders. 
Theorem 33. There exists an absolutely $\boldsymbol{\Delta}_{2}^{\mathbf{1}}$ reduction from isomorphism on codes for countable ordinals to isomorphism on vertex-transitive linear orders.

Proof. The aim is to show that there exists a $\boldsymbol{\Delta}_{2}^{\mathbf{1}}$ map from codes for ordinals $\alpha$ to codes for $\mathbb{Z}^{\alpha}$. We can construct such a map by recursion on $\alpha$. Given a code $<_{L}$ on $\omega$ for $L$ we define, for $n, m \in L \cdot \mathbb{Z}$ where $n=\left\langle n_{0}, n_{1}\right\rangle$ and $m=\left\langle m_{0}, m_{1}\right\rangle, n<_{L \cdot \mathbb{Z}} m$ if and only if $n_{0}<_{\mathbb{Z}} m_{0}$ or $n_{0}=m_{0}$ and $n_{1}<_{L} m_{1}$. For limit stages, given a code for a limit ordinal $\lambda$, together with a $\lambda$-sequence of codes for $\mathbb{Z}^{\alpha}, \alpha<\lambda$, we can produce a code for $\mathbb{Z}^{\lambda}=\left(\sum\left\{\mathbb{Z}^{\gamma} \cdot \omega \mid \gamma<\lambda\right\}\right)^{*}+1+\sum\left\{\mathbb{Z}^{\gamma} \cdot \omega \mid \gamma<\lambda\right\}$ in a similar manner.

It is not difficult to check that each step of this recursive construction is Borel. Further, it is well known that this implies we can construct a code for $\mathbb{Z}^{\beta}$ in an absolutely $\boldsymbol{\Delta}_{2}^{\mathbf{1}}$ fashion. For example, an infinite time turing machine (ITTM) can easily be programmed to carry out the recursive construction, and ITTM-computable mappings are always absolutely $\boldsymbol{\Delta}_{\mathbf{2}}^{\mathbf{1}}$ (for the definition of ITTM and the statement of this fact, see [5]). 


\section{CHAPTER 4}

\section{VERTEX-TRANSITIVE TOURNAMENTS}

We now shift focus to the classification problem for vertex-transitive tournaments. As tournaments are a broader class than linear orders, but still a subset of the class of directed graphs it makes sense to consider the complexity of these structures as well. While the exact complexity of vertex-transitive tournaments is yet to be determined, the main result in this chapter states that isomorphism of vertex-transitive tournaments is properly more complex than $E_{0}$, or eventual equality on $2^{\omega}$. A remaining question is to determine whether this is, in fact, Borel complete.

Definition 34. A tournament is a directed graph in which every pair of vertices is connected by an edge.

Recall that a tournament is vertex-transitive if its automorphism groups acts transitively on the set of vertices. We denote the isomorphism relation over vertextransitive tournaments as $\cong_{V T T}$.

Further, we note the following two relations which will be necessary in the proof of the main theorem. First, eventual equality on $2^{\omega}$ is denoted $E_{0}$. More formally, we say

$$
x E_{0} y \Leftrightarrow \exists m \forall n \geq m x(n)=y(n)
$$


We will find that, instead of working with $E_{0}$ directly, we will need to work with a subset of the domain of $E_{\mathbb{Z}}$, or the shift equivalence on $2^{\mathbb{Z}}$. That is,

$$
x E_{\mathbb{Z}} y \Leftrightarrow \exists m \forall n x(n+m)=y(n)
$$

In order to show that isomorphism of vertex-transitive tournaments is more complex than $E_{0}$ we will see that the necessary reduction only works on a subset of the domain of $E_{\mathbb{Z}}$. However, this makes it necessary to check that the restriction of $E_{\mathbb{Z}}$ to this subset remains as complex as $E_{0}$ which leads us to the following proposition.

Proposition 35. $E_{0}$ on $2^{\omega}$ is Borel bireducible to $E_{\mathbb{Z}} \uparrow_{\mathcal{C}}$ on $2^{\mathbb{Z}}$ for any comeager set C.

Proof. First, we claim that $E_{\mathbb{Z}}$ has a dense orbit. To see this, note that $2^{\mathbb{Z}}$ has the topology with basic open sets $V_{t}=\left\{x \in 2^{\mathbb{Z}} \mid t \subset x\right\}$. Recall that $2^{<\omega}$ is countable and let $\left\{s_{i}\right\}_{i \in \omega}$ enumerate $2^{<\omega}$. Further, we define $x=\oslash \frown s_{1} \frown s_{2} \frown \cdots \frown s_{i} \frown \cdots$ where $\oslash$ denotes the sequence of all 0 on $\omega^{*}$. This $x$ is certainly in $2^{\mathbb{Z}}$ and contains an instance of every finite binary sequence. That is, given $s \in 2^{<\omega}$, there exists some $i$ such that $s=s_{i}=x(j)\left\lceil_{\left|s_{i}\right|}\right.$ where $\left|s_{i}\right|$ denotes the length of the sequence $s_{i}$.

Further, given $s \in 2^{<\omega}$ as before, we know that $s \subset z \cdot x$ as $s=x(j-z)\lceil|s|$. Therefore, shifts of $x$ also contain an instance of every finite binary sequence.

The aim is to show that, given $[x]_{\mathbb{Z}}=\{z \cdot x \mid z \in \mathbb{Z}\}$ where $z \cdot x=x(n+z)$ for all $n \in \mathbb{Z}$, every $V_{t}$ contains an element of $[x]_{\mathbb{Z}}$.

That is, given any $V_{t}$, we know that $t \subset z \cdot x$ for some $z \in \mathbb{Z}$ and so $t \in[x]_{\mathbb{Z}}$. Therefore $t \in V_{t} \cap[x]_{\mathbb{Z}}$ and so there exists a dense orbit. As this is the case, we know

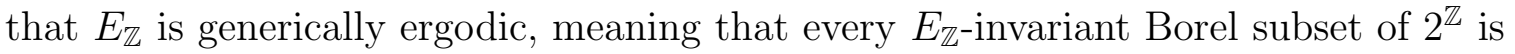
either meager or comeager (Proposition 6.1.9, [4]). 
As $E_{\mathbb{Z}}$ is generically ergodic, we also know that $E_{\mathbb{Z}} \Upsilon_{\mathcal{C}}$ is generically ergodic (Proposition 6.1.9, [4]). As this is the case and all orbits of $\mathbb{Z}$ are countable, hence meager, we find that $E_{\mathbb{Z}} \Upsilon_{\mathcal{C}}$ is not smooth (Proposition 6.1.10, [4]). As $E_{\mathbb{Z}} \Upsilon_{\mathcal{C}}$ is not smooth, one can conclude that $E_{0}$ is Borel reducible to $E_{\mathbb{Z}} \Upsilon_{\mathcal{C}}$ (Proposition 6.3.1, [6]). On the other hand, we know that any $\mathbb{Z}$-orbit relation is Borel reducible to $E_{0}[2]$ and therefore $E_{Z} \uparrow_{\mathcal{C}}$ is Borel bireducible with $E_{0}$.

Thus we arrive at the main result for vertex-transitive tournaments. That is, the isomorphism relation for vertex-transitive tournaments is more complex than $E_{0}$.

Theorem 36. There exists a Borel reduction from $E_{0}$ to isomorphism of vertextransitive tournaments.

Proof. Given $x \in 2^{\mathbb{Z}}$, we construct a tournament, $T_{x}$, with vertices in $\mathbb{Z} \times \mathbb{Z}$ as follows.

Given two vertices at positions $(m, n)$ and $\left(m^{\prime}, n^{\prime}\right)$ where $m, n, m^{\prime}, n^{\prime} \in \mathbb{Z}$, we define

$$
(m, n) \rightarrow\left(m^{\prime}, n^{\prime}\right) \Leftrightarrow\left\{\begin{array}{l}
m=m^{\prime} \text { and } n>n^{\prime} \text { or } \\
m^{\prime}=m+1 \text { and } x\left(n^{\prime}-n\right)=1 \text { or } \\
\left|m-m^{\prime}\right| \geq 2 \text { and } m<m^{\prime}
\end{array}\right.
$$

where, otherwise, there is an edge $\left(m^{\prime}, n^{\prime}\right) \rightarrow(m, n)$. An example of this can be seen in the figure below, where we focus on a pair of columns which are "one apart".

Note that $x$ is bi-infinite, but for illustration purposes we highlight five consecutive terms in $x$ and the five corresponding vertices in relation to vertex $(0,0)$.

It is important to note that these $T_{x}$ are, in fact, vertex-transitive as the automorphism carrying the vertex at position $(0,0)$ to position $(m, n)$ is given by the map $(a, b) \mapsto(a+m, b+n)$ preserves the definition of edge direction given above. 


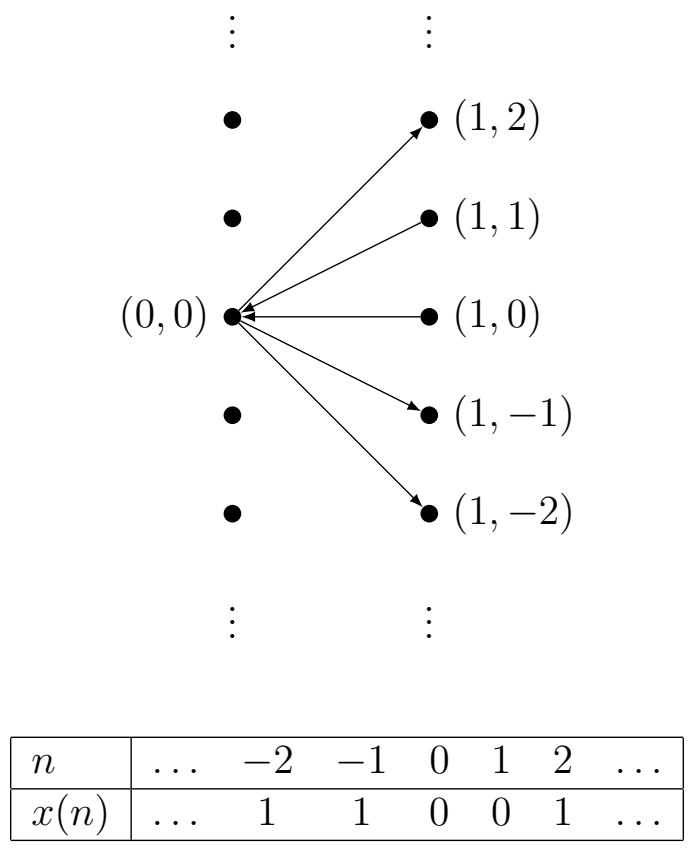

Figure 4.1: Directed edges from vertex $(0,0)$ to vertices one column to the right.

Now that we have developed the construction of $T_{x}$ given $x$, the aim is to show that there exists a comeager subset $\mathcal{C} \subseteq 2^{\mathbb{Z}}$, which will be defined later, such that, for all sequences $x, x^{\prime} \in \mathcal{C}$,

$$
x E_{\mathbb{Z}} x^{\prime} \Leftrightarrow T_{x} \cong T_{x^{\prime}}
$$

To begin, suppose that $x E_{\mathbb{Z}} x^{\prime}$. That is, there exists some $k \in \mathbb{Z}$ such that, for every $n \in \mathbb{Z}, x(n)=x^{\prime}(n+k)$. Now, construct two vertex-transitive tournaments, $T_{x}$ and $T_{x^{\prime}}$, from $x$ and $x^{\prime}$ respectively as described above. In order to see that $T_{x}$ and $T_{x^{\prime}}$ are isomorphic, choose any column in $T_{x}$ and similarly for $T_{x^{\prime}}$. We claim that $\varphi(m, n)=(m, n+k m)$ is an isomorphism between $T_{x}$ and $T_{x^{\prime}}$.

To see this, note that $\varphi$ sends $m$ to $m$ and $n$ to $m+k n$, both of which are linear, so 
we certainly have that $\varphi$ is bijective. Further, we notice that adjacency of vertices is preserved as, if $(m, n) \rightarrow\left(m^{\prime}, n^{\prime}\right)$ in $T_{x}$ then we claim that $(m, n+k m) \rightarrow\left(m^{\prime}, n^{\prime}+k m^{\prime}\right)$ in $T_{x}^{\prime}$. To check this, we refer to the above cases where $(m, n) \rightarrow\left(m^{\prime}, n^{\prime}\right)$.

If $m=m^{\prime}$ and $n>n^{\prime}$ then $\varphi(m, n) \rightarrow \varphi\left(m^{\prime}, n^{\prime}\right)$ as $m=m^{\prime}$ and so $n+k m>$ $n^{\prime}+k m^{\prime}$. If $m+1=m^{\prime}$ and $x\left(n^{\prime}-n\right)=1$ then we arrive at the same conclusion as $m+1=m^{\prime}$ and $x^{\prime}\left(\left(n^{\prime}+k m^{\prime}\right)-(n+k m)\right)=x^{\prime}\left(n^{\prime}-n+k\right)=x\left(n^{\prime}-n\right)=1$. Finally, if $\left|m-m^{\prime}\right| \geq 2$ and $m<m^{\prime}$ then it still remains that $\varphi(m, n) \rightarrow \varphi\left(m^{\prime}, n^{\prime}\right)$ as $\varphi$ leaves $m$ and $m^{\prime}$ unchanged. Thus, we are able to conclude that $\varphi: T_{x} \rightarrow T_{x}^{\prime}$ is an isomorphism.

For the other direction, consider $T_{x} \cong T_{x^{\prime}}$. That is, $\varphi: T_{x} \rightarrow T_{x^{\prime}}$ gives an isomorphism as described. To see that $x E_{\mathbb{Z}} x^{\prime}$, we must first see that we can recover $x$ from $T_{x}$. In order to do so, we require that $x$ have sufficiently many 0 and 1 values. To be precise, we require at least one of the following conditions:

(i) For every $n$ there exists some $k<n$ such that $x(k-n)=1$ and $x(k)=0$

(ii) For every $n$ there exists some $k$ such that $x(-k)=0$ and $x(k-n)=0$

Note that, excluding the trivial case where $w=(-1, n)$, each of these conditions on $x$ are $G_{\delta}$ and dense, hence comeager. With these requirements, we now come to the definition of the comeager set $\mathcal{C}$ containing all $x \in 2^{\mathbb{Z}}$ satisfying the above conditions.

By the above conditions, for any vertex $v$, we can identify the five columns surrounding $v$ which we will denote as $S_{v}$. More specifically, $S_{v}$ is the set of points involved in a three-cycle with $v$.

To see this, let $v=(0,0)$ and consider a second vertex $w$. In the case that $w=(0, n), n<0$ is in the same column as $v$ then condition (i) ensures that there is a third vertex $u$ such that $w \rightarrow u$ and $u \rightarrow v$. Condition (i) ensures the same in 
the case that $w=(-1, n)$ or $w=(1, n), n \in \mathbb{Z}$. On the other hand, if $w=(-2, n)$ or $w=(2, n)$ then condition (ii) ensures that there exists a third vertex $u$ such that $w \rightarrow u$ and $u \rightarrow v$

We note that it is impossible for there to be a three-cycle involving two vertices that are more than two columns apart. To see that this is the case, suppose that $v=(0,0)$ and $w=(m, n)$ for $|m| \geq 3$ and for some $n \in \mathbb{Z}$. Further, suppose there is a third vertex $u$ such that $w \rightarrow u$. If $m \leq-3$ then $w \rightarrow v$ and, by assumption $w \rightarrow u$. Therefore $w$ cannot be involved in a three-cycle with $v$. So now, if we consider $m \geq 3$, we certainly have $v \rightarrow w$. Since $w \rightarrow u$ then it must be that $m^{\prime} \geq m-1$ and so $m^{\prime} \geq 2$. That is, $\left|0-m^{\prime}\right|=\left|m^{\prime}\right| \geq 2$ and so $v \rightarrow u$. Once again, we see that $w$ cannot be involved in a three-cycle with $v$ based on our construction.

Further, we can determine distinct columns within $S_{v}$ as follows:

(i) Let $C_{v}$ denote the set of all vertices $w \in S_{v}$ such that $S_{w}=S_{v}$

(ii) Let $C_{-2, v}$ denote the set of all vertices $w \in S_{v}$ such that $v$ does not arrow anyone in $C_{w}$

(iii) Let $C_{2, v}$ denote the set of all vertices $w \in S_{v}$ such that $v$ is in $C_{-2, w}$

(iv) Let $C_{-1, v}$ denote the set of all vertices $w \in S_{v}$ such that $w \notin C_{-2, v}$ and every $z \in C_{2, v}$ is contained in $S_{w}$

(v) Let $C_{1, v}$ denote the set of all vertices $w \in S_{v}$ such that for every $z \in C_{-1, v}$, $w \in C_{2, z}$.

Focusing on $C_{1, v}$, we can recover the sequence $x$ by choosing any $v$ and looking at edges between $v$ and each $w \in C_{1, v}$. If we repeat this process for $T_{x^{\prime}}$ we then 
have $x$ and $x^{\prime}$. Well, as $\varphi$ is an isomorphism between $T_{x}$ and $T_{x^{\prime}}, \varphi$ must map $C_{1, v}$ (a $\mathbb{Z}$-ordered subgraph) in $T_{x}$ to $C_{1, \varphi(v)}$ in $T_{x^{\prime}}$ in an order preserving way. The only possible map is a shift. That is, the edges from $v$ to vertices in $C_{1, v}$ in $T_{x}$ are a shift of the edges from $\varphi(v)$ to vertices in $C_{1, \varphi(v)}$ in $T_{x^{\prime}}$. Well, as $x$ and $x^{\prime}$ were used to define these edges respectively it must be the case that $x$ is a shift of $x^{\prime}$ and so $x E_{\mathbb{Z}} x^{\prime}$ as expected.

Finally, we note that the $\cong_{V T T}$ is strictly more complex than $E_{0}$. That is, we recall the existence of a $\Delta_{2}^{1}$ reduction from $E_{\omega_{1}}$ to the isomorphism relation on vertextransitive linear orders. As linear orders are tournaments we also get that there is a $\Delta_{2}^{1}$ reduction from $E_{\omega_{1}}$ to the isomorphism relation on vertex-transitive tournaments. If it were the case that $E_{0} \sim_{B} \cong_{V T T}$ then there would exist a Borel reduction from $\cong_{V T T}$ to $E_{0}$. But that would mean $E_{\omega_{1}}$ was reducible to $E_{0}$ which cannot be the case as $E_{0}$ and $E_{\omega_{1}}$ are incomparable (refer to the remark following Corollary 3.3 in [7]) and so we reach a contradiction. 


\section{REFERENCES}

[1] John D. Clemens. Isomorphism of homogeneous structures. Notre Dame Journal of Formal Logic, 50(1):1-22, 2009.

[2] R. Dougherty, S. Jackson, and A. S. Kechris. The structure of hyperfinite Borel equivalence relations. Trans. Amer. Math. Soc., 341(1):193-225, 1994.

[3] Harvey Friedman and Lee Stanley. A Borel reducibility theory for classes of countable structures. J. Symbolic Logic, 54(3):894-914, 1989.

[4] Su Gao. Invariant descriptive set theory, volume 293 of Pure and Applied Mathematics (Boca Raton). CRC Press, Boca Raton, FL, 2009.

[5] Joel David Hamkins and Andy Lewis. Infinite time Turing machines. J. Symbolic Logic, 65(2):567-604, 2000.

[6] L. A. Harrington, A. S. Kechris, and A. Louveau. A Glimm-Effros dichotomy for Borel equivalence relations. J. Amer. Math. Soc., 3(4):903-928, 1990.

[7] Greg Hjorth. An absoluteness principle for Borel sets. J. Symbolic Logic, 63(2):663-693, 1998.

[8] Alan H. Mekler. Stability of nilpotent groups of class 2 and prime exponent. $J$. Symbolic Logic, 46(4):781-788, 1981. 
[9] Joseph G. Rosenstein. Linear orderings, volume 98 of Pure and Applied Mathematics. Academic Press, Inc. [Harcourt Brace Jovanovich, Publishers], New York-London, 1982. 